\title{
Stimulating Innovations in the Measurement of Parenting Constructs
}

\author{
Louise C. Mâsse, $\mathrm{PhD}^{\prime}$ and Allison W. Watts, MSc ${ }^{2}$
}

\section{Abstract}

Parents can play a crucial role in the development of children's behaviors associated with dietary habits, physical activity, and sedentary lifestyles. Many parenting practices and/or styles measures have been developed; however, there is little agreement as to how the influence of parenting should be measured. More importantly, our ability to relate parenting practices and/or styles to children's behaviors depends on its accurate assessment. While there is a need to standardize our assessment to further advance knowledge in this area, this article will discuss areas that may stimulate advances in the measurement of parenting constructs. Because self-report measures are important for the assessment of parenting, this article discusses whether solutions to improve selfreport measures may lie in: (1) Improving the questions asked; (2) improving the methods used to correct for social desirability or measurement errors; (3) changing our measurement paradigm to assess implicit parenting behaviors; (4) changing how self-report is collected by taking advantage of ecological momentary assessment methods; (5) using better psychometric methods to validate parenting measures or alternatively using advances in psychometric methods, such as item banking and computerized adaptive testing, to solve common administration issues (i.e., response burden and comparability of results across studies); and (6) employing novel technologies to collect data such as portable technologies, gaming, and virtual reality simulation. This article will briefly discuss the potential of technologies to measure parenting constructs.

\section{Introduction}

$\mathbf{T}$ he familial environment can play a crucial role in the prevention and treatment of childhood obesity. Parents can set the stage for the development of healthy behaviors by influencing their child's preferences, shaping their environment, and acting as agents of change. ${ }^{1-7}$ The importance of the familial environment for both the prevention and treatment of childhood obesity is widely recognized ${ }^{1,7}$; however, the scientific evidence is weak, especially for the treatment of obesity where reviews have yielded varied support (i.e., supporting evidence ${ }^{3,8}$ versus weak to no evidence $\left.{ }^{7,9}\right)$. In part, this may be explained by the psychometric properties of measures used to assess parenting and the considerable variation as to how parenting is assessed across studies. ${ }^{7,10}$ A recent position statement by the American Heart Association calls for better measures of parenting to further understand the mechanisms of influence on children's weight-related behaviors [i.e., poor dietary habits, lack of physical activity (PA), and sedentary lifestyles]. ${ }^{7}$
Parents are thought to influence their child's behavior through two distinct, but interrelated, constructs - general parenting style and specific parenting practices. General parenting style reflects the parental attitudes and beliefs that create the broad emotional climate in which parentchild interactions take place. ${ }^{11-13}$ Parenting style is commonly described by typologies that classify parents based on their levels of responsiveness and demandingness, with the most common classifications being authoritarian, authoritative, permissive, and neglectful. ${ }^{14,15}$ Distinct from parenting style, parenting practices are the specific strategies that parents use to achieve desired outcomes. ${ }^{11-13}$ Numerous measures have been developed to assess parenting styles and practices; however, there is little consistency among them or agreement as to how parenting should be measured.

With the exception of observational studies, ${ }^{16,17}$ selfreport questionnaires dominate how weight-related parenting constructs are typically assessed. ${ }^{11,18}$ Because questionnaire data continue to be an important methodology in the assessment of parenting, more attention should

\footnotetext{
'Department of Pediatrics/School of Population and Public Health, University of British Columbia, Vancouver, BC, Canada.

${ }^{2}$ School of Population and Public Health, University of British Columbia, Vancouver, BC, Canada.

The abstract was presented at the preconference to the 2012 International Society for Behavioral Nutrition and Physical Activity (ISBNPA) annual meeting, Houston, TX, May 2012.
} 
focus on addressing many of the methodological concerns associated with self-report. ${ }^{11,19}$ In an attempt to address some of these methodological concerns, this article will discuss whether potential solutions lie in: (1) Improving how the questions are asked, (2) Improving the methods used to correct for social desirability and measurement error, (3) changing our measurement paradigm, (4) changing how self-report is collected, (5) Using better psychometric methods, and (6) using novel technologies as alternative data collection methods. This article aims to stimulate innovations in the measurement of parenting by briefly discussing approaches that may be used; however, those interested in recommendations for the field should consult other articles published within this supplement. ${ }^{20-23}$

\section{Potential Solutions for Improving Parenting Assessment}

\section{Improving Questions Asked}

Developing a good survey question requires the consideration of many factors that have been thoroughly discussed in measurement textbooks (e.g., ambiguity, double-barreled questions). ${ }^{24}$ Nevertheless, a greater understanding of how question formats influence social desirability for parenting measures is largely unknown. ${ }^{25}$ Morsbach has suggested four formatting strategies to consider for improving the measurement of parenting constructs: (1) Normalizing formats that use preamble statements to make all responses to a question acceptable and normal (e.g., parents view opposing statements related to family breakfast practices, "some families regularly eat breakfast together" versus "some families rarely eat breakfast together," and indicate which statement is a common practice for their family); (2) positive framing formats that focus on asking about behaviors that elicit less socially desirable responses (e.g., asking a parent about their level of activity is more positive and may elicit less social desirability than asking about their TV viewing behavior); (3) supportive or "saving face" formats that serve to normalize a given behavior and thus elicit more truthful answers (e.g., a question worded: "I usually enroll my children in physical activity programs, but this time I did not" versus "I do not enroll my children in physical activity programs" may decrease perceived judgment when agreeing to the statement); and (4) time-use question formats that ask parents to list the activities that they engaged in over a given time period instead of responding directly about participation in a specific activity. ${ }^{25}$ Support for these four formatting strategies has been assessed in a few studies and in other behavioral contexts (e.g., sexual behavior, physical activity, voting, and volunteering). ${ }^{26-29}$ The utility of these formatting strategies to decrease social desirability of weight-related parenting constructs requires further investigation to determine whether such approaches reduce social desirability or if some formats (i.e., positive framing) elicit different recall that are still subject to social desirability. ${ }^{24}$

\section{Correcting for Social Desirability} and Measurement Errors

Social desirability scales were initially developed to identify and correct for a respondent's biased response style; that is, a conscious attempt to portray oneself in a positive light. ${ }^{24}$ While socially desirable responding likely occurs with self-report, these scales (e.g., MarloweCrowne Social Desirability Scale, ${ }^{30}$ Balanced Inventory of Desirable Responding, ${ }^{31}$ Social Desirability Scale- $17^{32}$ ) have perhaps not lived up to their expectations. ${ }^{33-35}$ Despite wide use, there is little evidence that correcting for social desirability can help decrease measurement errors associated with self-report. ${ }^{33-35}$ In addition, absence of a correlation with a self-report measure may not eliminate the possibility of social desirability bias, while correcting for a correlation has also resulted in misleading findings and unwarranted reduction in power. ${ }^{34}$

As the utility of social desirability scales continues to be debated, ${ }^{33-35}$ the method comparison approach (measurement error models), commonly employed to correct for measurement error in dietary assessment, holds promise. ${ }^{36,37}$ This approach has the potential to account for both social desirability bias as well as other sources of measurement error. With respect to parenting, the method comparison approach can be used to compare self-report to a different data collection method such as observation, indirect questioning, or a corroborating source. Observations in the home setting can provide an opportunity to monitor parent behaviors that can be compared to selfreport data. It is important to note that certain parenting practices may be particularly difficult to observe unobtrusively as they occur with less frequency. ${ }^{38}$ In addition, the utility of observations is limited for older children, who interact less frequently with parents and are more reactive to the observations. ${ }^{38}$ Nonetheless, this approach may have utility for certain parenting practices, such as mealtime feeding behaviors. ${ }^{38}$ Alternatively, data collected through indirect questioning can be compared to self-report because it may elicit more truthful information on sensitive issues. ${ }^{33,39}$ Finally, corroborating data collected from another source (e.g., spouse) can serve to cross-validate the self-report data; evidence from addiction research suggests parents may respond more accurately if they believe corroborating evidence is collected. ${ }^{40}$ However, these sources may also be biased given their close association with the respondent. ${ }^{33}$ While there are limitations to measurement error models, they are becoming more established and can be used to correct for measurement error associated with parenting measures. ${ }^{33,37,41}$

Structural equation modeling can provide an alternative approach to account for measurement error. For example, this is achieved by treating all survey items measuring parenting as a latent construct, meaning that the items are aggregated as one factor. Measurement error can then be estimated by taking into account the correlations among the individual items. As a result, the association between 
the parenting measures and children's weight related behavior is disattenuated, meaning that the estimated association accounts for measurement error. ${ }^{33}$

\section{Expanding Our Measurement Paradigm}

Implicit measures and social desirability. Advances in social cognition research suggest that "implicit" measures can be useful in assessing socially desirable behaviors. ${ }^{42-44}$ Self-report or explicit measures are typically operationalized to ask questions closely linked to the behavior(s) assessed, thus making the respondent fully aware of the cognitive or affective tasks measured. ${ }^{42}$ In contrast, implicit measures assess a given construct without asking direct questions and sometimes without the participant's knowledge of what is being measured by the test. ${ }^{44}$ Selfreport or explicit measures have been considered problematic when assessing socially desirable constructs since the respondents can reflect on how they will answer the question. ${ }^{43}$ In a recent meta-analysis, the predictive validity of explicit measures decreased sharply when used to assess socially desirable constructs while this was not the case for implicit measures. ${ }^{42}$ In addition, the incremental predictive validity of implicit measures improved when socially desirable constructs were measured, providing evidence that both explicit and implicit measures are independently associated with behaviors. ${ }^{42}$ Although the utility of implicit measures is not without controversy (e.g., reliability averaging 0.56 and predictive validity varying across domains), ${ }^{42,45}$ the extent to which such measures can improve the assessment of parenting measures, assumed to be affected by social desirability, is largely unknown.

Implicit measures and impulsivity. Besides removing social desirability bias, implicit measures are believed to tap into impulsive influence, a dimension not currently captured with self-report measures. ${ }^{46,47}$ Although the development of implicit measures has been somewhat atheoretical, the utility of expanding measures of parenting to assess both reflective and impulsive influences may best be grounded by reviewing the basic tenets of Dual-Process Theories. ${ }^{47}$ Dual-Process Theories assume that two distinct processes regulate health behaviors, namely: (1) Reflective influences (measured with self-report or explicit measures) and (2) impulsive influences (measured with implicit measures). ${ }^{46,47}$ On the one hand, it is assumed that behavioral goals or desired parenting practices require conscious or reflective thought processes. ${ }^{46,47}$ However, it is acknowledged that impulsive influences may override desired goal-directed behaviors. Certain stimuli in the environment (e.g., under increased cognitive load) may activate more reactive or impulsive behaviors. ${ }^{46,47}$ For example, after a stressful day, parents may be unable to override less desirable practices, such as letting their child eat in front of the TV. Impulsivity has been acknowledged in proposed modifications of existing health behavior theories and has been incorporated in self-report or explicit measures by making the questions more context specific. ${ }^{46}$ Proponents of implicit measures, however, believe the assessment methods should minimize the interference of conscious control in order to tap into the part of the brain where impulsive behavioral schema are stored. ${ }^{46}$

As a result, measuring impulsive behaviors is believed to be possible only through implicit measures. ${ }^{46}$ Many health behavior theories/models assume that higher-order processes regulate health behavior; therefore, it seems reasonable that reflective and impulsive behaviors employ fundamentally different processes worthwhile of measurement. ${ }^{46}$ The extent and frequency with which reflective or impulsive parenting practices are activated is unclear and likely varies among individuals and contexts. As the science of implicit measures gains empirical evidence, ${ }^{42}$ it might be worthwhile to further understand how impulsive influences regulate parenting practices.

Developing implicit measures of parenting. Developing implicit measures for the assessment of parenting may be challenging because it is not clear how such measures would be operationalized. Currently, implicit measures are often used to understand the attitude-behavior relationship; however, they are also used to measure other constructs including, among others, personality traits, alcohol and drug use, clinical phenomena, and relationships. ${ }^{42}$ The Implicit Association Test (IAT) is the most widely used implicit method, although other methods exist (e.g., sequential priming procedures, Go/No-go Association Task, Sorting Paired Features task, Brief Implicit Association Test). ${ }^{47-50}$ The IAT uses timed association tasks to tap into unconscious or automatic social cognitive processes presumed to assess implicit behavior toward the construct of interest. ${ }^{42,47}$ For example, the weight stigma IAT uses pictures of overweight and normal weight individuals (targeted construct) and combines them with positive and negative attributes (e.g., bad vs. good). ${ }^{51}$ When the targeted construct is paired with the attributes that match the participant's attitude, their response time is expected to be faster. For example, participants with faster response times when the overweight pictures are combined with negative attributes would exhibit implicit bias against overweight individuals. ${ }^{51}$ For a demonstration of this measure and other IAT measures, see the Project Implicit ${ }^{\circledR}$ website. $^{52}$ Although the utility of the IAT method to measure parenting constructs may be questioned, implicit measures have the potential to address issues of social desirability and capture impulsive influences related to parenting, making such an approach worthwhile to investigate.

\section{Changing How Self-Report Is Collected}

Ecological Momentary Assessment. Ecological Momentary Assessment (EMA) or real-time data capture can provide an alternative method to collect self-report data. EMA consists of collecting repeated data in the natural 
environment, where the behavior is experienced. ${ }^{53}$ For example, in studies where participants report their physical activity, respondents are periodically prompted by a computer to indicate whether they were physically active that day. ${ }^{54}$ The data are typically collected over a number of days to get a representative sampling of the respondent's level of physical activity. ${ }^{54}$ EMA data collection techniques have moved from paper-and-pencil to electronic diaries, palm pilot devices, computer tablets, and mobile phones. ${ }^{55}$ The utility of EMA has improved with the advancement of technology because it can both cue the respondent to collect data as well as provide a device for collecting the data. ${ }^{56}$ EMA has been successful in assessing many health behaviors (e.g., drug use/abuse, tobacco use, physical activity, diet), and there is a growing body of evidence supporting the predictive validity of these assessments. ${ }^{53}$ Potential advantages of EMA include assessing behaviors in the moment and in the social and environmental context in which the behaviors occur; therefore, producing more ecologically valid data that are less influenced by recall bias. ${ }^{53,57}$

The procedure is not without limitations. For example, the utility of the method may be influenced by compliance and adherence to the data collection protocol because it results in missing data that poses analytical challenges, the intensive nature of the method may potentially influence behavior (measurement reactivity), and the complexity in processing the data may impede its uses despite advancements in multilevel analytical models that facilitate data processing. ${ }^{58}$ Nonetheless, EMA can assess fluctuation in behaviors over time, providing an opportunity to assess exposure. Exposure is a concept derived from environmental sciences ${ }^{59}$ that can serve to assess the extent to which parenting practices are sustained or fluctuate over a period of time.

Viewing parenting from an exposure perspective can be particularly relevant as the stability of some parenting measures has been low. ${ }^{60}$ EMA offers the advantage of collecting data over a period of time to measure actual fluctuations in parenting practices in an attempt to quantify exposure. In the context of cardiovascular disease risk factors, for example, Kamarck showed that sustained exposure to psychological stress at work, measured with EMA, was associated with cardiovascular risk progression, whereas the standard self-report was not associated. ${ }^{59}$ Fluctuations in parenting practices seem likely to occur, therefore it appears that EMA may be best at quantifying which practices children are exposed to and, in particular, to assess fluctuations in parenting practices. In addition to assessing exposure, EMA provides an opportunity to measure the dynamic behavioral processes that may be particularly important for the assessment of parenting. ${ }^{59}$

EMA also moves the assessment of parenting into dynamic assessment models that provide more information about within-person variation. From a health behavior modification perspective, measuring dynamic behavioral processes can lead to testing new hypotheses related to behavior change. ${ }^{61}$ In the context of mobile health interventions, Riley discussed how our current health behavior theories or models fall short on integrating "intraindividual dynamic regulatory processes." 61 Specifically, current theories or models do not integrate mechanisms to explain intraindividual variations nor do they incorporate feedback loops. ${ }^{61}$ Therefore, refining current health behavior theories or models would be essential to guide the operationalization of parenting measures with the EMA methodology. For parenting, EMA can assess dual dynamic processes because it provides the opportunity to examine both variations within parent and child and how these variations interact. The parent-child interaction will likely add to the complexity of using EMA in parenting research but may elucidate mechanisms of behavior not previously examined.

\section{Using Better Psychometric Methods}

The utility of Item Response Theory (IRT). Numerous articles have demonstrated the utility and advantages of modern psychometric methods (e.g., Item Response Theory (IRT) $)^{62-64}$; however, these methods are seldom, if ever, employed to evaluate the psychometric properties of parenting measures. IRT analyses provide a different way of examining item properties as the rules of measurement are different under these procedures when compared to Classical Test Theory (CTT) methods. ${ }^{65,66}$ A few of these properties are highlighted below because they have interesting applications for improving the measurement of parenting constructs with a more detailed discussion presented in Embretson. ${ }^{66}$ First, an interesting difference between IRT and CTT is that the item statistics (i.e., item discrimination or item difficulty) are invariant, meaning that they do not depend on the variance of a given sample. For example, the item discrimination index (i.e., assessed with the item-total correlation with CTT) is not influenced by the variance of the sample for IRT item statistics. As a result, the psychometric properties of a parenting construct would remain stable across populations. Second, the CTT reliability index (e.g., Cronbach alpha) is closely tied to the number of items included in a scale and, as a result, longer scales are generally more reliable. Under some circumstances IRT can result in more reliable scores with shorter scales, thus, serving to decrease response burden. Third, given the invariance properties of the items, it is easier to compare scores on a similar construct that is measured with different items with IRT equating methodologies. ${ }^{62,65,67,68}$ The properties of IRT make it easier to modify measures across administrations/studies and to incorporate developmentally appropriate items while ensuring that the results are comparable across administrations/studies. Most importantly, IRT provides the foundation for shortening parenting measures. Although there are other advantages of using IRT to evaluate the psychometric properties of a scale, the primary benefit is reduced response burden through measurement administration while maintaining 
comparability across studies. For these reasons, the field would benefit from using Computerized Adaptive Testing (CAT) methodologies to administer parenting measures.

CAT administrations. CAT has been widely used in the educational context for collecting patient reported outcomes, such as the NIH Patient Reported Outcomes Measurement Information System (PROMIS ${ }^{\circledR}$ ) initiative. ${ }^{69}$ CAT methods are well developed and provide the option of tailoring the administration of self-report questionnaires. Unlike other administration methods, CATs are dynamic assessments where the response to an initial item influences which items the computer will select in the item bank. Following each response, an estimate of the respondent's score is computed. If the score is precise enough, determined a priori based on a desired confidence interval, the administration is stopped. Alternatively, if the score is not precise enough, more items will be administered. ${ }^{69,70}$ For patient-reported outcome measures, the NIH PROMIS $^{\circledR}$ initiative has been able to demonstrate that CAT administrations can result in more precise, valid, and reliable scores with fewer items (e.g., 4 to 8 items) administered per construct measured. ${ }^{68,69,71}$ CAT administrations can be advantageous for the assessment of parenting because the intent is to administer as few items as possible while maintaining the precision, validity, and reliability of the scores without sacrificing the ability to compare scores across studies.

The process established by the NIH PROMIS ${ }^{\circledR}$ initiative to develop CAT measures for the assessment of patientreported outcomes can serve as a model for developing CAT measures of parenting. ${ }^{72}$ Until recently, CAT administrations have typically required the use of proprietary software, limiting their use; however, the NIH PROMIS ${ }^{\circledR}$ initiative has created the Assessment Center ${ }^{\mathrm{SM}}$, which provides a platform for administering measures with CAT while allowing further development of the measures by researchers. ${ }^{73}$ Clearly, the methodology and procedures for administering measures with CAT are well established and can be easily employed for assessing parenting.

To take advantage of CAT administrations for the assessment of parenting, item banks (repository of calibrated items) need to be developed. An item bank provides the necessary foundation for developing CAT administrations as well as supports the development of tailored measures (allowing researchers to select the items) or fixed-length short forms. ${ }^{68}$ Developing an item bank requires a rigorous process that typically involves multiple steps similar to the ones employed for developing a measure. ${ }^{74}$ Specifically, it involves: (1) Creating an operational definition of the construct to be measured achieved by reviewing the literature (i.e., reviewing existing measures) and getting input from experts and perspectives from participants; (2) identifying existing items, often achieved by conducting a search of the scientific literature and reviewing existing measures; (3) binning, winnowing, and supplementing the items, where binning refers to classifying the items into the appropriate domain assessed, winnowing consists of eliminating redundant items or poorly written items, and supplementing consists of adding items to ensure appropriate content coverage; (4) revising and standardizing the items to ensure the items pooled from various sources have similar styles and response formats; (5) conducting a qualitative review of the items with experts and participants; and (6) assessing the psychometric properties of the items as well as calibrating them. ${ }^{74,75}$ Involving the broader scientific community in this process would bring more attention to the developmental stages of a measure, which is essential for establishing the validity of parenting measures.

\section{Using Novel Technologies}

Growing advances in technology have led to significant changes in the assessment of health behaviors. Dietary assessment can now be assessed with an Automated SelfAdministered 24- Hour Dietary Recall. ${ }^{76,77}$ In addition, integration of cell phone and digital imaging, ${ }^{78,79}$ as well as wearable camera and voice recognition, has been developed to collect dietary intake. ${ }^{80}$ Laboratory experiments have been conducted to show that multiple sensors (e.g., accelerometers, infrared and ultraviolet light, and global positioning systems) located on different body parts can measure physical activity or sedentary behaviors unobtrusively in real-life settings. ${ }^{81,82}$ Clearly, application of technology is rapidly changing the assessment of health behaviors, but the extent to which applications of technology can be used to improve the assessment of parenting is yet to be seen. Observations of parent behavior have been used to study feeding practices by video recording family mealtime. ${ }^{83,84}$

As technologies are becoming smaller, portable, and less intrusive, it is possible to extend observation to many interactions outside of mealtimes. The extent to which observational methods can be used with older children, who interact less frequently with parents and are more reactive to observation, is unclear. ${ }^{38}$ In addition, the extent that technology will facilitate the assessment of interactions related to physical activity practices is less clear, because these interactions likely occur in less defined time frames than mealtime feeding behaviors. Although technology can improve observational assessment, there is a need to consider whether technology can be used in new ways to "objectively" assess parenting constructs since current methods (i.e., self-report and observations) have inherent limitations.

The methods employed to measure health behaviors can certainly inspire innovations in the measurement of parenting as can those employed to deliver health interventions where technology has been widely embraced. The proliferation of advanced information and communication technology has changed the delivery mode of interventions (e.g., internet, personal digital assistant, and mobile phones) and how the interventions are presented, 
such as using text, sound, and video, employing animations, or using simulated experiences (e.g., games, virtual realities). ${ }^{85,86}$ Many of these delivery modes have been used with self-report. However, the extent to which animations or simulated experiences may provide an alter- native mode of data collection for assessing parenting constructs has yet to be explored. In the virtual world, researchers have found that adopting an avatar identity (i.e., taking on a graphical character of oneself) can modify how people interact in their virtual environments and make

\section{Table I. List of Potential Approaches or Studies That May Be Used To Improve Measures}

\section{of Parenting}

\section{Areas}

Improving questions asked
Examples of studies or approaches that may be used to improve measures of parenting

Conduct cognitive interviews to identify response patterns in response to various item formats.

Examine response patterns from various item formats to determine the effect of social desirability.

Examine whether the strength and direction of the association between parenting practices and children's weight-related behaviors is affected when various item formats are employed.

Correcting for social desirability and measurement error

Determine the potential of measurement error models by: (I) Determining which comparison methods (e.g., observational, alternative data collection methods, or corroborating sources) can be used with different parenting constructs and (2) testing whether these approaches improve associations between parenting practices and children's weight-related behaviors.

Use structural equation modeling (or other appropriate statistical methods) to estimate disattenuated associations between parenting practices and children's weight-related behaviors (i.e., association corrected for measurement errors).

Expanding our measurement paradigm

Work collaboratively with social cognition researchers to develop and validate implicit measures of parenting.

Conduct comparative analyses to examine the extent to which implicit measures versus current measures of parenting are associated with children's weight-related behaviors to determine whether implicit measures improve these associations.

Changing how self-report is collected

Develop data collection protocols to determine the feasibility of collecting parenting behaviors with Ecological Momentary Assessment (EMA). Examine what data collection protocols yield high compliance and adherence as well as minimize reactivity.

Conduct longitudinal studies to examine fluctuations in parenting practices across behaviors to determine which "parenting practices" would be better assessed with EMA versus "standard" self-report.

Develop and validate algorithms for quantifying parenting practice(s) as exposure variable(s).

Determine whether the strength of the association between parenting practices and children's weight related behavior is stronger when assessed with EMA versus with "standard" self-report.

Expand current health behavior theories / models by elaborating on the dynamic mechanisms that can be tested with EMA to simultaneously assess parenting practices and children's weight related behaviors.

Test dynamic behavioral processes of how parenting practices influence children's weight related behaviors over time and vice versa.

Using better psychometric methods

Standardize the conceptual definitions and content of parenting measures to facilitate comparison across studies.

Work collaboratively to develop calibrated/validated item banks for the assessment of parenting practices to ensure measures can be administered in a variety of ways: (I) Short fixed forms; (2) tailored forms; and (3) computerized adaptive testing (CAT) forms.

Develop an infrastructure, modeled after or integrated into the NIH PROMIS ${ }^{\circledR}$ initiative, ${ }^{69}$ to allow researchers to freely access item bank measures as well as to have an interface to administer these parenting measures using CAT.

Establish standards and processes for translating and validating the item banks in other languages.

Examine the external validity of the item bank(s) administered with CAT to determine the extent to which the CAT methodology improves the strength of the associations between parenting practices and children's weight related behaviors.

Develop and validate the methodology to integrate CAT administration in the EMA context.

Using novel technologies
Take advantage of portable technologies to assess parenting practices in the natural environment to either validate self-report data or as a primary source of data collection.

Determine whether alternatives to self-report can be used to collect valid and reliable data about parenting practices, such as data collected in the gaming or virtual world contexts. 
them more likely to divulge sensitive behaviors. ${ }^{87,88}$ This highlights the utility of the virtual world to assess socially desirable behaviors such as parenting. In addition, the virtual world can: (1) Simulate conditions that resemble the real world setting, such as food shopping in a virtual store $^{89,90}$; (2) enable the creation of scenarios that are standardized across subjects ${ }^{88}$; and (3) elicit both explicit and implicit behavior, since nonverbal cues can be assessed with an array of technology (e.g., eye tracking). ${ }^{88}$ Virtual reality has been used in other contexts to measure behaviors including food purchasing behaviors. ${ }^{89,90}$ In addition, it has been used to assess weight bias among medical students in treating nonobese and obese patients. ${ }^{88}$ Technology continues to be used in novel ways in research, and the utility of virtual realities or developing games for assessing parenting styles or practices remains to be explored.

\section{Conclusion}

This article discussed a number of solutions to improve measures of parenting. This article is part of a larger supplement on this topic, and recommendations for the field are published in other articles that are part of this supplement. ${ }^{20-23}$ The extent to which certain areas might be more worthwhile to explore than others remains unknown. To help researchers move the field forward in each of the areas discussed, Table 1 lists strategies that may be employed or studies that may be conducted to address each of the areas brought forward in this article. Table 1 is not meant to be comprehensive, but it highlights how one can begin to improve measures of parenting.

Although not discussed in this article, some of the strategies presented are clearly more appropriate to integrate in small-scale studies than in large epidemiological studies. The assessment of parenting is further complicated, for example, by differences that occur based on familial configuration (e.g., multigenerational household, shared custody arrangements, same sex parents), parentchild relationships, and when older or younger siblings are present. To take into account the complexities of the modern family environment, advancements will need to be made not only to our measurement strategies but also to our analytical approaches.

Currently, much attention has been focused on how parents and the family environment influence childhood obesity. There remain significant concerns surrounding the measures used to assess parenting constructs, thus making it difficult to examine the influence of parenting on children's weight related behaviors. Creating better measures is the foundation to better science; therefore, concerted efforts are needed to rethink how best to operationalize measures of parenting in order to move the field forward.

\section{Acknowledgments}

The preconference to the 2012 International Society for Behavioral Nutrition and Physical Activity (ISBNPA) annual meeting, "Parenting Measurement: Current Status and Consensus Reports" and resulting manuscripts were made possible due to funding from the United States Department of Agriculture/Agricultural Research Service (USDA/ARS 2012-68001-19285) and the National Heart, Lung, and Blood Institute of the National Institutes of Health (R13HL114262). L.C.M. was funded through salary support provided by the Michael Smith Foundation for Health Research, the Child and Family Research Institute, and the Sunny Hill foundation. A.W.W. was funded by the Joint Canadian Institutes of Health Research (CIHR) and Heart and Stroke Foundation of Canada Training Grant in Population Intervention for Chronic Disease Prevention: A Pan-Canadian Program (Grant \#: 53893) and a Doctoral Research Award from CIHR and the Danone Institute of Canada.

\section{Author Disclosure Statement}

No competing financial interests exist.

\section{References}

1. Summerbell CD, Waters E, Edmunds LD, et al. Interventions for preventing obesity in children. Cochrane Database Syst Rev $2007 ; 1-70$.

2. Golley RK, Hendrie GA, Slater A, et al. Interventions that involve parents to improve children's weight-related nutrition intake and activity patterns - what nutrition and activity targets and behaviour change techniques are associated with intervention effectiveness? Obes Rev 2011;12:114-130.

3. Skouteris H, McCabe M, Swinburn B, et al. Parental influence and obesity prevention in pre-schoolers: A systematic review of interventions. Obes Rev 2011;12:315-328.

4. Kitzmann KM, Beech BM. Family-based interventions for pediatric obesity: Methodological and conceptual challenges from family psychology. J Fam Psychol 2006;20:175-189.

5. Nowicka P, Flodmark CE. Family in pediatric obesity management: A literature review. Int J Pediatr Obes 2008;3(Suppl 1):44-50.

6. Epstein LH, Paluch RA, Roemmich JN, et al. Family-based obesity treatment, then and now: Twenty-five years of pediatric obesity treatment. Health Psychol 2007;26:381-391.

7. Faith MS, Van HL, Appel LJ, et al. Evaluating parents and adult caregivers as "agents of change" for treating obese children: Evidence for parent behavior change strategies and research gaps: A scientific statement from the American Heart Association. Circulation 2012;125:1186-1207.

8. Summerbell CD, Ashton V, Campbell KJ, et al. Interventions for treating obesity in children (review). Cochrane Database Syst Rev 2007;1-57.

9. McGovern L, Johnson JN, Paulo R, et al. Clinical review: Treatment of pediatric obesity: A systematic review and meta-analysis of randomized trials. J Clin Endocrinol Metab 2008;93:46004605.

10. Pritchett R, Kemp J, Wilson P, et al. Quick, simple measures of family relationships for use in clinical practice and research. A systematic review. Fam Pract 2011;28:172-187.

11. Sleddens EF, Gerards SM, Thijs C, et al. General parenting, childhood overweight and obesity-inducing behaviors: A review. Int $J$ Pediatr Obes 2011;6:e12-e27. 
12. Hennessy E, Hughes SO, Goldberg JP, et al. Parent-child interactions and objectively measured child physical activity: A crosssectional study. Int J Behav Nutr Phys Act 2010;7:71.

13. Stewart SM, Bond MH. A critical look at parenting research from the mainstream: Problems uncovered while adapting Western research to non-Western cultures. Br J Dev Psychol 2002;20:379-392.

14. Baumrind D. Current patterns of parental authority. Dev Psychol 1971;4:1-103.

15. Maccoby EE, Martin JA. Socialization in the context of the family: Parent-child interaction. In: Mussen PH, Hetherington EM (eds), Handbook of Child Psychology: Socialization, Personality, and Social Development. Wiley \& Sons, Inc.: New York, 1983, pp. 1-101.

16. Robertson AS, Rivara FP, Ebel BE, et al. Validation of parent self reported home safety practices. Inj Prev 2005;11:209-212.

17. Hawes D, Dadds M. Assessing parenting practices through parentreport and direct observation during parent-training. J Child Fam Stud 2006;15:554-567.

18. van der Horst K, Oenema A, Ferreira I, et al. A systematic review of environmental correlates of obesity-related dietary behaviors in youth. Health Educ Res 2007;22:203-226.

19. Walker LO, Kirby RS. Conceptual and measurement issues in early parenting practices research: An epidemiologic perspective. Matern Child Health J 2010;14:958-970.

20. Davison KK, Masse L, Timperio A, et al. Physical activity parenting measurement and research: Challenges, explanations, and solutions. Child Obes 2013;9(S1):S-103-S-109.

21. Power TG, Sleddens EFC, Berge J, et al. Contemporary research on parenting: Conceptual, methodological, and translational issues. Child Obes 2013;9(S1):S-87-S-94.

22. Hughes SO, Frankel LA, Beltran A, et al. Food Parenting measurement issues: Working Group Consensus Report. Child Obes 2013;9(S1):S-95-S-102.

23. O'Connor TM, Hingle M, Chuang R-J, et al. Conceptual understanding of screen media parenting: Report of a Working Group. Child Obes 2013;9(S1):S-110-S-118.

24. Streiner DL, Norman GR. Health Measurement Scales: A Practical Guide to Their Development and Use. Oxford University Press: Oxford, 2008.

25. Morsbach SK, Prinz RJ. Understanding and improving the validity of self-report of parenting. Clin Child Fam Psychol Rev 2006;9:1-21.

26. Catania J, Binson D, Canchola J, et al. Effects of interviewer gender, interviewer choice, and item wording on responses to questions concerning sexual behavior. Public Opin Q 1996;60:345-375.

27. DiFranceisco W, McAuliffe T, Sikkema K. Influences of survey instrument format on the reliability of self-reported high risk sexual behavior. AIDS Behav 1998;2:329-337.

28. Belli R, Traugott M, Young M, et al. Reducing vote overreporting in surveys: Social desirability, memory failure, and source monitoring. Public Opin Q 1999;63:90-108.

29. Presser S, Stinson L. Data collection mode and social desirability bias in self-reported religious attendance. Am Sociol Rev 1998;63:137-145.

30. Crowne DP, Marlowe D. A new scale of social desirability independent of psychopathology. J Consult Psychol 1960;24:349-354.

31. Paulhus D. Two-component models of socially desirable responding. J Pers Soc Psychol 1984;46:598-609.

32. Stöber J. The Social Desirability Scale-17 (SDS-17): Convergent validity, discriminant validity, and relationship with age. Eur $J$ Psychol Assess 2001;17:222-232.

33. Jo M. Controlling social-desirability bias via method factors of direct and indirect questioning in structural equation models. Psychol Market 2000;17:137-148.
34. Leite WL, Beretvas SN. Validation of scores on the MarloweCrowne Social Desirability Scale and the Balanced Inventory of Desirable Responding. Educ Psychol Meas 2005;65:140-154.

35. Uziel L. Rethinking social desirability scales: From impression management to interpersonally oriented self-control. Perspect Psychol Sci 2010;5:243-262.

36. Kipnis V, Freedman LS, Brown CC, et al. Effect of measurement error on energy-adjustment models in nutritional epidemiology. Am J Epidemiol 1997;146:842-855.

37. Buonaccorsi JP. Measurement Error: Models, Methods, and Applications. Chapman \& Hall/CRC Press: Boca Raton, FL, 2010.

38. Shelton K, Frick P, Wootton J. Assessment of parenting practices in families of elementary school-age children. J Clin Child Psychol 1996;25:317-329.

39. Fisher R. Social desirability bias and the validity of indirect questioning. J Consum Res 1993;20:303-315.

40. Del Boca FK, Noll JA. Truth or consequences: The validity of selfreport data in health services research on addictions. Addiction 2000;959(Suppl 3):S347-S360.

41. Nusser SM, Beyler NK, Welk GJ, et al. Modeling errors in physical activity recall data. J Phys Act Health 2012;9(Suppl 1):S56-S67.

42. Greenwald AG, Poehlman TA, Uhlmann EL, et al. Understanding and using the Implicit Association Test: III. Meta-analysis of predictive validity. J Pers Soc Psychol 2009;97:17-41.

43. Ham J, van den Bos K. On unconscious and conscious thought and the accuracy of implicit and explicit judgments. Soc Cognition 2011;6:648-667.

44. Fazio RH, Olson MA. Implicit measures in social cognition. research: Their meaning and use. Annu Rev Psychol 2003;54:297-327.

45. Nosek BA, Smyth FL. A multitrait-multimethod validation of the Implicit Association Test: Implicit and explicit attitudes are related but distinct constructs. Exp Psychol 2007;54:14-29.

46. Hofmann W, Friese M, Wiers RW. Impulsive versus reflective influences on health behavior: A theoretical framework and empirical review. Health Psychology Rev 2008;2:111-137.

47. Nosek BA, Hawkins CB, Frazier RS. Implicit social cognition: From measures to mechanisms. Trends Cogn Sci 2011;15:152-159.

48. Greenwald AG, McGhee DE, Schwartz JL. Measuring individual differences in implicit cognition: The implicit association test. J Pers Soc Psychol 1998;74:1464-1480.

49. Nosek BA, Greenwald AG, Banaji MR. Understanding and using the Implicit Association Test: II. Method variables and construct validity. Pers Soc Psychol Bull 2005;31:166-180.

50. Bar-Anan Y, Nosek BA, Vianello M. The sorting paired features task: A measure of association strengths. Exp Psychol 2009;56: 329-343

51. Schwartz MB, Chambliss HO, Brownell KD, et al. Weight bias among health professionals specializing in obesity. Obes Res 2003;11:1033-1039.

52. Project Implicit ${ }^{\circledR}$. Available at https://implicit.harvard.edu/implicit/. Last accessed on May 15, 2013.

53. Shiffman S. Ecological momentary assessment (EMA) in studies of substance use. Psychol Assess 2009;21:486-497.

54. Dunton GF, Intille SS, Wolch J, et al. Investigating the impact of a smart growth community on the contexts of children's physical activity using Ecological Momentary Assessment. Health Place 2012;18:76-84.

55. Ferguson SG, Shiffman S. Using the methods of ecological momentary assessment in substance dependence research - smoking cessation as a case study. Subst Use Misuse 2011;46:87-95. 
56. Stone AA, Shiffman S, Atienza AA, et al. Historial roots and rationale of ecological momentary assessment (EMA). In: Stone AA, Shiffman S, Atienza AA, and Nebeling L, (eds), The Science of Real-Time Data Capture: Self-Reports in Health Research. Oxford University Press: New York, 2007, pp. 3-10.

57. Heron KE, Smyth JM. Ecological momentary interventions: Incorporating mobile technology into psychosocial and health behaviour treatments. Br J Health Psychol 2010;15:1-39.

58. Myin-Germeys I, Oorschot M, Collip D, et al. Experience sampling research in psychopathology: Opening the black box of daily life. Psychol Med 2009;39:1533-1547.

59. Kamarck TW, Muldoon MF, Shiffman S, et al. Experiences of demand and control in daily life as correlates of subclinical carotid atherosclerosis in a healthy older sample. Health Psychol 2004;23: 24-32.

60. Cullen KW, Baranowski T, Rittenberry L, et al. Child-reported family and peer influences on fruit, juice and vegetable consumption: Reliability and validity of measures. Health Educ Res 2001;16:187-200.

61. Riley WT, Rivera DE, Atienza AA, et al. Health behavior models in the age of mobile interventions: Are our theories up to the task? Transl Behav Med 2011;1:53-71.

62. Mâsse LC, Allen D, Wilson M, et al. Introducing equating methodologies to compare test scores from two different self-regulation scales. Health Educ Res 2006;21(Suppl 1):i110-i120.

63. Mâsse LC, Wilson M, Baranowski T, et al. Improving psychometric methods in health education and health behavior research. Health Educ Res 2006;21(Suppl 1):i1-i3.

64. Mâsse LC, Heesch KC, Eason KE, et al. Evaluating the properties of a stage-specific self-efficacy scale for physical activity using classical test theory, confirmatory factor analysis and item response modeling. Health Educ Res 2006;21(Suppl 1):i33-i46.

65. Embretson SE, Reise SP. Item Response Theory for Psychologists. Lawrence Erlbaum Associates, Publishers: Mahwah, NJ, 2000.

66. Embretson SE. The new rules of measurement. Psychol Assessment 1995;8:341-349.

67. Van der Linden WJ, Hambleton RK. Handbook of Modern Item Response Theory. Springer-Verlag: New York, 1997.

68. Forrest CB, Bevans KB, Tucker C, et al. Commentary: The Patient-Reported Outcome Measurement Information System $\left(\right.$ PROMIS $^{\circledR}$ ) for children and youth: Application to pediatric psychology. J Pediatr Psychol 2012;37:614-621.

69. Cella D, Yount S, Rothrock N, et al. The Patient-Reported Outcomes Measurement Information System (PROMIS): Progress of an NIH Roadmap cooperative group during its first two year. Med Care 2007;45:S3-S11.

70. Lai JS, Cella D, Choi S, et al. How item banks and their application can influence measurement practice in rehabilitation medicine: A PROMIS fatigue item bank example. Arch Phys Med Rehabil 2011;92:S20-S27.

71. Yost KJ, Eton DT, Garcia SF, et al. Minimally important differences were estimated for six Patient-Reported Outcomes Measurement Information System-Cancer scales in advanced-stage cancer patients. J Clin Epidemiol 2011;64:507-516.

72. Revicki DA, Cella DF. Health status assessment for the twentyfirst century: Item response theory, item banking and computer adaptive testing. Qual Life Res 1997;6:595-600.

73. Gershon R, Rothrock NE, Hanrahan RT, et al. The development of a clinical outcomes survey research application: Assessment Center. Qual Life Res 2010;19:677-685.
74. DeWalt DA, Rothrock N, Yount S, et al. Evaluation of item candidates: The PROMIS qualitative item review. Med Care 2007;45: S12-S21.

75. Christodoulou C, Junghaenel DU, DeWalt DA, et al. Cognitive interviewing in the evaluation of fatigue items: Results from the patient-reported outcomes measurement information system (PROMIS). Qual Life Res 2008;17:1239-1246.

76. Subar AF, Thompson FE, Potischman N, et al. Formative research of a quick list for an automated self-administered 24-hour dietary recall. J Am Diet Assoc 2007;107:1002-1007.

77. Thompson FE, Subar AF, Loria CM, et al. Need for technological innovation in dietary assessment. J Am Diet Assoc 2010;110:48-51.

78. Sun M, Fernstrom JD, Jia W, et al. A wearable electronic system for objective dietary assessment. J Am Diet Assoc 2010;110:45-47.

79. Six BL, Schap TE, Zhu FM, et al. Evidence-based development of a mobile telephone food record. J Am Diet Assoc 2010;110:74-79.

80. Weiss R, Stumbo PJ, Divakaran A. Automatic food documentation and volume computation using digital imaging and electronic transmission. J Am Diet Assoc 2010;110:42-44.

81. Intille SS, Lester J, Sallis JF, et al. New horizons in sensor development. Med Sci Sports Exerc 2012;44:S24-S31.

82. Intille SS, Albinali F, Mota S, et al. Design of a wearable physical activity monitoring system using mobile phones and accelerometers. Conf Proc IEEE Eng Med Biol Soc 2011;2011:3636-3639.

83. Haycraft E, Blissett J. Eating disorder symptoms and parenting styles. Appetite 2010;54:221-224.

84. Worobey J, Lopez MI, Hoffman DJ. Maternal behavior and infant weight gain in the first year. J Nutr Educ Behav 2009;41:169-175.

85. Lau PW, Lau EY, Wong del P, et al. A systematic review of information and communication technology-based interventions for promoting physical activity behavior change in children and adolescents. J Med Internet Res 2011;13:e48.

86. Johnston JD, Massey AP, De Vanneaux C. Innovation in weight loss interventions programs: An examination of a 3D virtual world approach. International Conference on System Sciences 2012;2890-2899.

87. Gay G, Pollak J, Adams P, et al. Pilot study of Aurora, a social, mobile-phone-based emotion sharing and recording system. J Diabetes Sci Technol 2011;5:325-332.

88. Persky S. Application of virtual reality methods to obesity prevention and management research. J Diabetes Sci Technol 2011; 5:333-339.

89. Ruppert B. New directions in the use of virtual reality for food shopping: marketing and education perspectives. J Diabetes Sci Technol 2011;5:315-318.

90. Burke R. Virtual shopping: Breakthrough in marketing research Harvard Bus Rev 1996;74:120-131.

Address correspondence to:

Louise C. Mâsse, PhD Associate Professor Department of Pediatrics/School of Population and Public Health

University of British Columbia

F508-4480 Oak Street

Vancouver, BC, Canada V6H 3V4

E-mail: 1masse@cfri.ubc.ca 